Kompass

Pneumologie

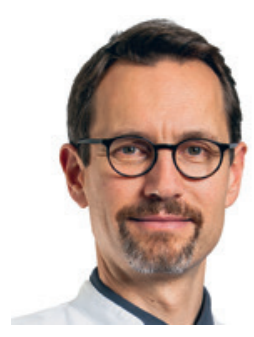

\section{Gernot Rohde}

Universitätsklinikum Frankfurt, Medizinische Klinik I, Pneumologie/Allergologie, Frankfurt am Main, Deutschland

\title{
Präzisionsmedizin: Pneumologie im Wandel
}

Liebe Kolleginnen und Kollegen, liebe Leserinnen und Leser,

das Fokusthema des vorliegenden Heftes lautet passend zum Motto des diesjährigen DGP-Kongresses «Präzisionsmedizin: Pneumologie im Wandel», welcher pandemiebedingt virtuell im Juni stattfinden und den Fokus auf prognostisch relevante Fortschritte der Pneumologie in der personalisierten Präzisionsmedizin richten wird.

Die Medizin differenziert und (unter-)spezialisiert sich im Großen und Ganzen zunehmend, ganz besonders auch das Fachgebiet der Pneumologie. Dieser Umstand stellt uns Pneumologen vor die Schwierigkeit, den Überblick über das gesamte diagnostische und therapeutische Spektrum zu behalten und diese Errungenschaften auch am Patienten anwenden zu können. Denn wenn wir die wichtigen grundlagenwissenschaftlichen Fortschritte in der Medizin erfolgreich nutzen wollen, müssen wir uns auf einen größeren Zusammenhang einstellen. Personalisierte Präzisionsmedizin bedeutet daher nun, dass die Diagnose und die darauffolgende Therapie keine Entscheidung eines Einzelnen mehr sind, sondern auch Netzwerke und künstliche Intelligenz daran beteiligt sein werden. Dabei sollten wir immer darauf achten, dass künstliche Intelligenz uns Ärzte und andere Beschäftigte im Gesundheitswesen soweit wie möglich unterstützt, aber wir als Individuen nicht gleichzeitig zu «big data» abgewertet werden.

Ein weiterer wesentlicher Aspekt in diesem Zusammenhang ist die Internationalisierung der medizinischen Forschung, sie bedingt eine ausgeprägte Einschränkung auf möglichst einfache diagnostische und therapeutische Ansätze. Hier wird teilweise «Evidenz» auf dem kleinsten gemeinsamen Nenner geschaffen. National exzellent entwickelte Verfahren finden somit in Diagnostik und Behandlung unzureichend Berücksichtigung. Darüber hinaus steht die Komplexität des menschlichen Körpers und seiner Erkrankungen dazu im Gegensatz. In diesem Spannungsfeld bewegt sich also die personalisierte Präzisionsmedizin. Wie kein anderes Fach deckt die Pneumologie ein sehr breites Spektrum ab: Als Beispiel möchte ich die individuelle Auswahl von Inhalativa nennen, deren Zulassung meist auf randomisierten, kontrollierten Studien basiert. Die Studienpopulationen gleichen aber nicht unbedingt den Patienten in den Wartezimmern. Der Pneumologe muss letztendlich alle Informationen integrieren und zusammen mit den objektiven Erkenntnissen auch seine Erfahrung und subjektive Faktoren einbringen, um eine optimale Behandlung des Patienten persönlich und präzise zu gestalten. Auch
Prof. Dr. Gernot Rohde

Universitätsklinikum Frankfurt, Medizinische Klinik I

Pneumologie/Allergologie

Theodor-Stern-Kai 7, 60590 Frankfurt am Main, Deutschland gernot.rohde@kgu.de 
einige der in dieser Ausgabe versammelten Wissenstransfer-Beiträge widmen sich diesen Fragestellungen.

Der diesjährige Kongress soll Antworten und Hilfestellungen, konzeptionell, aber auch ganz praktisch liefern, wie beispielsweise die molekularen Therapien genetisch determinierter Erkrankungen wie der zystischen Fibrose. Hier sind zweifelsohne große prognostisch relevante Fortschritte erzielt worden. Dennoch zeigt nicht jeder Patient die Entwicklung, die theoretisch zu erwarten wäre. Welche anderen Faktoren spielen hier eine Rolle? Welche Informationen benötigen wir, um diese Therapien so effektiv wie möglich zu nutzen?

In diese Richtung geht auch die Übersichtsarbeit von José Luis Lopez-Campos und Kollegen in diesem Heft: Trotz der aktuellen Innovationen in der Behandlung der chronisch-obstruktiven Lungenerkrankung (chronic obstructive pulmonary disease, (OPD) wurden in der patientenzentrierten Medizin keine wesentlichen Fortschritte erzielt. Die Empfehlungen der aktuellen Leitlinien basieren auf den Durchschnittsergebnissen klinischer Studien, sodass die medizinische Praxis als «mittelwertbasiert» bezeichnet werden kann. Die Autoren um Lopez-Campos gehen daher auf die Haupt- dilemmata bei der Suche nach einem patientenzentrierten Ansatz für das COPD-Management ein und liefern Vorschläge, wie diese in einer einzigen, einfach anwendbaren Strategie kombiniert werden können.

Die zielgerichtete Therapie des Lungenkarzinoms und die Immunonkologie ermöglichen einen großen Schritt hin zu einer Transformation einer terminalen in eine chronische Erkrankung. Wir müssen auch hier nicht nur von den klinischen Studien, sondern vor allem auch aus der Praxis lernen, um zu verstehen, welche Patienten in welcher Therapiephase wie optimal zu führen sind.

Personalisierte Präzisionsmedizin bedeutet also auch ständiges Lernen aus der Praxis mit der Umkehr der Forschung «from bedside to bench». Es kommen folglich große Herausforderungen auf uns zu und der erfahrene Kliniker wird hierbei eine zunehmend wichtige Rolle einnehmen müssen: In Ihrer Fallserie berichten uns Philipp Eller und Kollegen von Patienten mit einer schweren Bindegewebserkrankung-assoziierten interstitiellen Lungenerkrankung und respiratorischer Insuffizienz. Bisher liegen aber noch keine randomisierten kontrollierten Studien oder Fall-Kontroll-Studien vor, die darauf schließen lassen, dass Im- munsuppressiva zur Behandlung dieser Patienten wirksam oder sicher ist. Dennoch konnten die Autoren nachweisen, dass die Betroffenen von einem aggressiven immunsuppressiven Therapieschema und einer extrakorporalen Blutreinigung mit schneller Verringerung der zirkulierenden Autoantikörper profitieren können.

Lassen Sie mich als letztes Beispiel die neue Kenntnis pulmonaler Infektionen anführen. Ausgangspunkt sind auch hier wesentliche technische Fortschritte, wie der molekulare Erregernachweis und das zunehmende Wissen um das Mikrobiom. Dabei stellen sich spannende Fragen, die bereits heute therapeutische Entscheidungen beeinflussen, zukünftig möglicherweise sogar entscheidend für das Verstehen chronischer (Lungen-)Erkrankungen sein werden.

Ich wünsche Ihnen viel Freude und Erkenntnisgewinn bei der Lektüre! Ihr

$$
1 \text { duth }
$$

Gernot Rohde

DGP-Kongresspräsident 2021 